\title{
Clinical Results with an Active Middle Ear Implant in the Oval Window
}

\author{
K.B. Hüttenbrink ${ }^{a} \cdot$ D. Beutner ${ }^{a} \cdot$ T. Zahnert $^{b}$

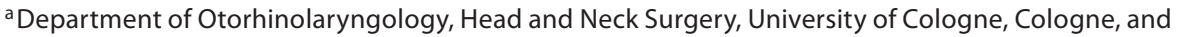 \\ ${ }^{b}$ Technical University of Dresden, Dresden, Germany
}

\begin{abstract}
Background: Some patients with chronic middle ear disease and multiple failed revisions, who also need a hearing aid, may benefit from an active middle ear implant. An advantage of an active middle ear implant is that the ear canal is unoccluded. Methods: Following extensive experimental development in temporal bones and investigations of various locations and attachments of a Vibrant Soundbridge transducer, a new titanium clip holder for the vibrant floating mass transducer was developed. This assembly is a total ossicular replacement prosthesis (TORP) that is placed on the stapes footplate. Six patients were implanted with this device. Results: Acoustic results demonstrate significantly improved gain, especially in the high frequencies, which is typically unobtainable by conventional hearing aids. Conclusion: The simple procedure of placing an active TORP assembly on the stapes footplate, similar to the implantation of a passive TORP prosthesis during tympanoplasty, offers promising treatment for cases of incurable middle ear disease.
\end{abstract}

Copyright $\odot 2010$ S. Karger AG, Basel

Patients with a long history of chronic otitis media often need a hearing aid for communication. This is not only due to unsuccessful restoration of acoustic function of the middle ear despite several tympanoplasty attempts, but also to an often accompanying inner ear hearing loss. Use of a conventional hearing aid ear mold may be uncomfortable because of surgically modified external ear anatomy.

After we developed a hydro-acoustic system that demonstrated the efficiency of direct vibratory stimulation of the inner ear via both round and oval windows [1], we tested the optimal placement of the commercially available Vibrant Soundbridge (VSB) (Vibrant MED-EL, Innsbruck, Austria) in temporal bone experiments. A titanium holder for the floating mass transducer (FMT) was developed in collaboration with the Kurz Company (Dusslingen, Germany). This total ossicular replacement prosthesis (TORP)-FMT assembly was implanted in 6 patients. 

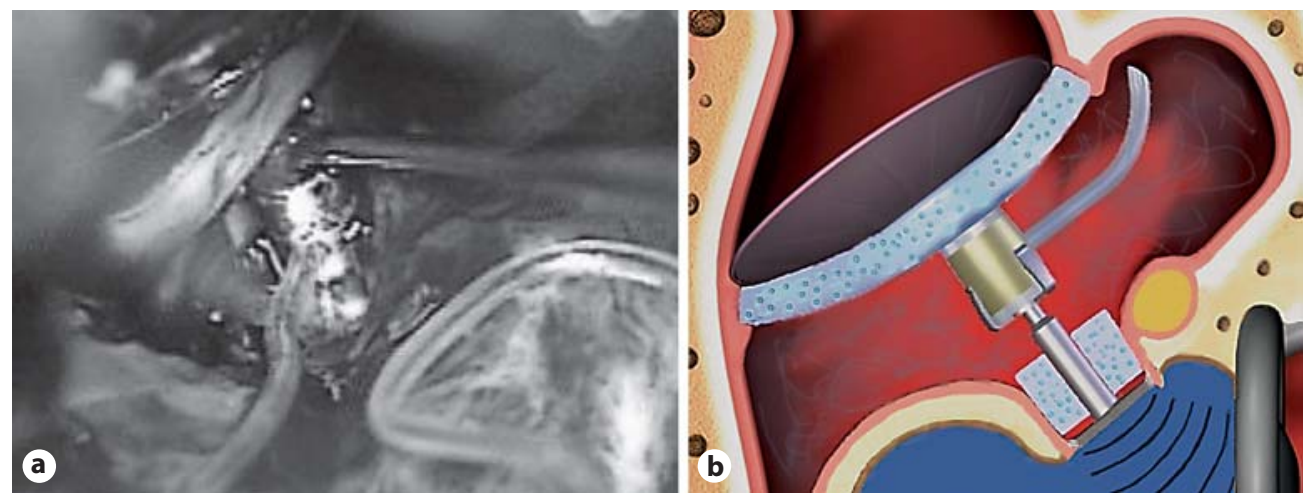

Fig. 1. a Intraoperative view of the assembly with the titanium holder and FMT in its clip, partly covered by the cartilage plate. $\mathbf{b}$ Schematic illustration of the TORP vibroplasty assembly in the oval window.

\section{Methods}

Six patients were implanted, 3 female and 3 male (mean age 67.5 years, range 61-75). The 2 right and 4 left ears had a severely destroyed middle ear with a bare footplate as the sole ossicular remnant, some (4 patients) with a nonfunctional passive TORP lying in the cavity. All patients had bilateral radical cavities with permanent air-bone gaps between 30 and $50 \mathrm{~dB}$ and significant (50 $\mathrm{dB}$ ) inner ear hearing losses. They did not tolerate conventional hearing aids.

After cleaning the oval window niche of granulations and scar tissue, we conducted reconstruction surgery. First, we prepared of a full thickness (1-mm) cartilage shoe with a central hole that was placed in the oval window niche. The rod of our new titanium support was inserted into the central hole and was centered on the footplate. The transducer was inserted into the support by gently pressing it down and fixing it between three clips (fig. 1). The top of the assembly was covered by a cartilage plate - the reconstructed tympanic membrane. In prior radical cavity obliteration cases, the cable was directed out behind the cartilage plates of the partial obliteration to the receiver in its separate bed in the temporalis squama.

\section{Results}

Audiometric thresholds, measured 4 weeks after surgery and after removal of ear packing, showed unchanged cochlear function. Figure 2 presents unaided bone conduction thresholds. Unaided air conduction thresholds remained nearly unchanged except in 1 patient, in whom it improved by $20 \mathrm{~dB}$ because the inactive assembly works as a passive TORP. Despite increased mass load and friction in the cartilage shoe holder, the average air conduction thresholds in the passive mode were comparable with the situation prior to implantation. 


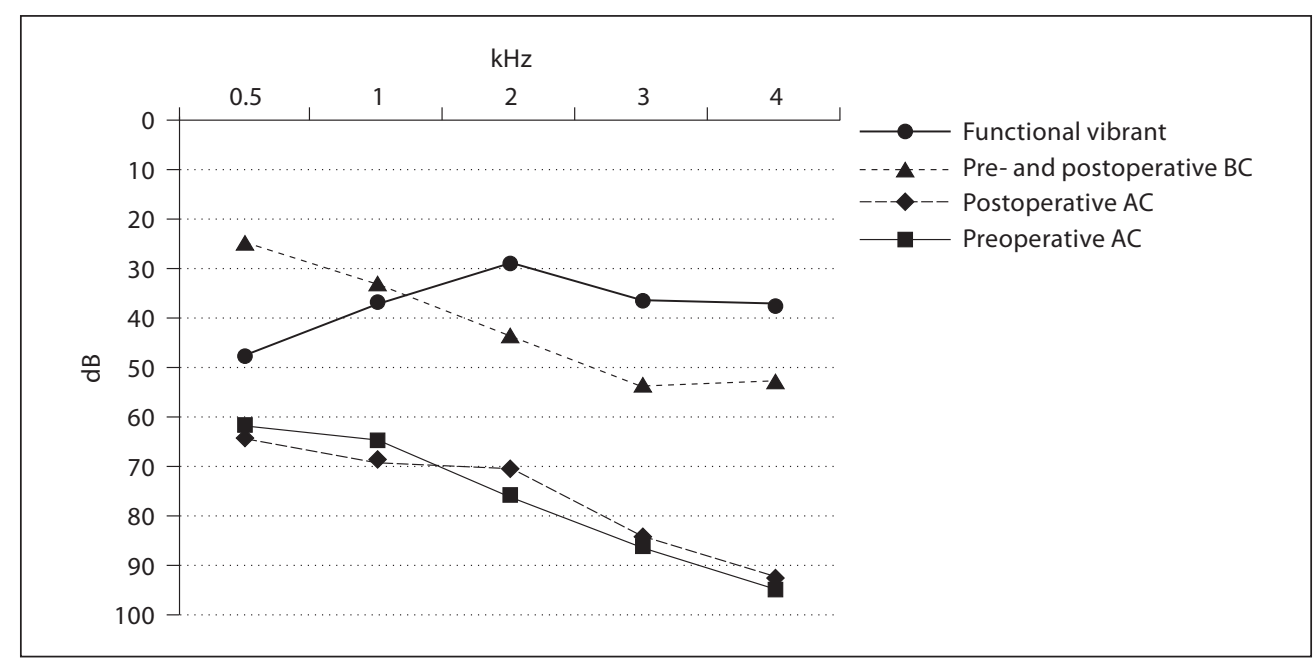

Fig. 2. Pre- and postoperative hearing thresholds of the first 6 patients demonstrating good gain at the high frequencies.

When the VSB was turned on, we observed an improvement of $20 \mathrm{~dB}$ in aided soundfield thresholds for frequencies 2 through $4 \mathrm{kHz}$ as compared with bone conduction thresholds (fig. 2).

Good high-frequency gain supported an improvement of monosyllabic word discrimination scores from $0 \%$ in the unaided condition to $55 \%$ in the VSB-aided condition (average scores, presentation level: $65 \mathrm{~dB}$, Freiburger monosyllabic word discrimination test). According to patients' reports, the improved high-frequency audibility between 2 and $4 \mathrm{kHz}$ was described as a more natural and very pleasant hearing sensation.

\section{Discussion}

Contrary to discussions on indications for implantable hearing aids in sensorineural hearing loss [2], their benefit in patients with permanently defect middle ear and unsatisfactory audiologic results with passive prostheses is demonstrated in an increasing number of successful implantations throughout the world [3-5]. The ear canal remains open, and the conductive component of the hearing loss is bypassed. Improvement in aided hearing thresholds, especially at high frequencies, is an important advantage. The alternative treatment, a bone-anchored hearing aid, is limited in cases of severe cochlear dysfunction because of limited amplification. For our patients, the surgical intervention was accepted because they had tolerated several revision tympanoplasties well in the past and had asked for additional revision tympanoplasty to improve their hearing. 
We analyzed the optimal position for the VSB FMT in the inner ear in our temporal bone experiments prior to clinical use [6]. Direct contact to the stapes footplate gives better performance, especially for high frequencies between 2 and $4 \mathrm{kHz}$ because of the large vibrating area of the complete footplate. Furthermore, the surgical procedure for the placement on the footplate might be seen as less demanding compared to the round window niche placement. Insertion into the round window niche often requires drilling the bony overhang with the risk of cochlear trauma [7]. This is due to restrictions in the approach, anatomical variations, and the relatively large diameter of the FMT $(1.6 \mathrm{~mm})$ as compared to the opening of the round window niche $(1.3-1.6 \mathrm{~mm})[8,9]$. Furthermore, permanently stable contact with the inner ear fluid, while also avoiding any hard contact to the surrounding bone, is mandatory for efficient coupling. A loose contact between the FMT and the round window membrane/ cochlear fluid results in reduced amplification. Mechanical restriction might explain the variable acoustic results reported in the literature on the round window placement. In some cases, the aided thresholds did not surpass the bone conduction thresholds [10].

Inconsistent contact of the FMT is avoided when the FMT is placed in direct contact with the footplate. Due to limited space in the oval window niche, a titanium holder was required. Secure attachment to the stapes footplate, preventing any lateral displacement, is assured when our cartilage shoe technique for the passive TORP procedure is used [11]. The FMT is attached to the support by modifying the clip design of our Clip-partial ossicular replacement prosthesis (PORP) [12]. In cases of an intact stapes, two clips (one for the FMT and the other for the stapes head) form the PORP design. Therefore, by using the developments for passive prostheses and by closing the tympanic cavity with a thick cartilage plate, stable assembly anchorage to the footplate is established.

Our first audiologic results support data gathered in our temporal bone experiments and demonstrate good acoustic benefit of directly coupling the FMT to the stapes footplate. Amplification of 40-60 dB in the high frequencies would not be possible with a conventional air conduction hearing aid. The surgical procedure, about as simple as the placement of a conventional TORP on the footplate, is another advantage over drilling the round window niche and the risk of inconsistent transducer placement in contact with the membrane.

Because the TORP also provides hearing benefit when used passively, patients with a moderate hearing loss might use active stimulation only in noisy or demanding listening situations (e.g. parties). Therefore, this TORP- (or future PORP-) supported VSB assembly can be offered to a large group of patients with permanently damaged middle ear function and additional sensorineural hearing loss, who are not candidates for conventional hearing aids or bone-anchored hearing aids. A good hearing prognosis can be offered to these patients with a simple and modified 'active tympanoplasty procedure'. 


\section{References}

1 Hüttenbrink KB: Biomechanical aspects in implantable microphones and hearing aids and development of a concept with a hydroacoustical transmission. Acta Otolarnygol 2001;121:185-189.

2 Hüttenbrink KB: Current status and critical reflexions on implantable hearing aids. Am J Otol 1999; 20:409-415.

3 Coletti V, Soli SD, Carner M, Colletti L: Treatment of mixed hearing losses via implantation of a vibratory transducer on the round window. Int J Audiol 2006;45:600-608.

4 Kiefer J: Round Window Stimulation with an Implantable Hearing Aid Soundbridge ${ }^{\circledR}$ combined with autogenous reconstruction of the auricle: a new approach. ORL 2006;68:375-385.

5 Wollenberg B: Integration of the active middle ear implant in total auricular reconstruction (in German). HNO 2007;55:349-356.

6 Hüttenbrink KB, Zahnert Th, Bornitz M, Beutner D: TORP-vibroplasty: a new alternative for the chronically disabled middle ear. Otol Neurotol 2008;29:965-971.
7 Pau HW, Just T, Bornitz M, Lasurashvilli M, Zahnert Th: Noise exposure of the inner ear during drilling a cochleostomy for cochlear implantation. Laryngoscope 2007;117:535-540.

8 Nomura Y: Otological significances of the round window. Adv Otorhinolaryngol 1984;33:11-99.

9 Roland MD: Cochlear implant electrode insertion: the round window revisited. Laryngoscope 2007; 117:1397-1402.

10 Soli S: Comparison of output levels and gains for bone conduction and round window stimulation of the cochlea in patients with conductive hearing loss. Proc 5th Int Symp on Human Sensibility Recovery Systems, Kyungpook National University Daegu, Korea, 2007.

11 Hüttenbrink KB, Zahnert T, Beutner D, Hofmann G: The cartilage guide: a solution for anchoring a columella prosthesis on footplate (in German). Laryngorhinootologie 2004;83:450-456.

12 Hüttenbrink KB, Zahnert Th, Wüstenberg E, Hofmann G: Titanium clip prosthesis. Otol Neurotol 2004;25:436-442.

Prof. Dr. K. B. Hüttenbrink

HNO-Uniklinik Köln

Kerpener Strasse 60

DE-50937 Köln (Germany)

Tel. +49 221478 4750, Fax +49 2214784793

E-Mail huettenbrink.k-b@uni-koeln.de 